\title{
Simulation of traffic lights for green wave and dynamic change of signal
}

\author{
Güney GORGUN, Ibrahim Halil GUZELBEY* \\ Department of Mechanical Engineering, University of Gaziantep, Gaziantep, Turkey \\ Email address: \\ gorgunguney@hotmail.com (G. GORGUN),guzelbeyih@gantep.edu.tr (I. H. GUZELBEY)
}

\section{To cite this article:}

Güney GORGUN, Ibrahim Halil GUZELBEY. Simulation of Traffic Lights for Green Wave and Dynamically Change of Signal. American Journal of Software Engineering and Applications. Vol. 2, No. 6, 2013, pp. 125-132. doi: 10.11648/j.ajsea.20130206.11

\begin{abstract}
In this study a traffic light system has been considered and simulated on Matlab to create hierarchical and logical model. This model is designed over five junctions to solve traffic jam in big cities by simulation of continuous flow of traffic lights. This simulation includes Green Wave flow and dynamic change of traffic lights due to change traffic volume. The simulation secures the continuous traffic flow by updating the light time for providing green wave flow.
\end{abstract}

Keywords: Matlab, Simulation, Traffic Lights, Green Wave Flow and Traffic Volume

\section{Introduction}

The history of traffic light starts with an idea of an engineer from, a Rail Way Engineer, John Peak Night, who specialized in designing signal systems for Britain's rail way. He realized no reason why these couldn't be adapted to roads. On 9 December 1868, the world's first traffic lights were set up at the junction of Bridge Street and George Street. Later on the first 4-way three color traffic lights were installed in 1920 in Detroit [1].

When it is investigated the technological development of traffic signal and control systems, it can be easily recognized remarkable improvement of signal systems. For example fuzzy systems deal with complex systems where imprecise, uncertain or ambiguous information is available, where the real world problems are nonlinear or where expert knowledge is expressed vaguely in natural language and fuzzy system was easily suited for traffic signal control. The general rule base for traffic signal control will develop with time, and also the fuzzy controller will become better with time [2-4]. Model formulation and solution of linear optimization problem for multiple intersections in a network was worked by explicit constraints capturing traffic movement on the links connecting the intersections [5].

Development technology has come a long way for detecting of cars in the traffic. It guides for organizing of traffic signal incase traffic congestion. For example AMR sensor (The anisotropic magneto resistive) is used for detection of ferrous object like cars [6]. In addition to them, an adaptive traffic control system was developed where the traffic load is continuously measured by loop detectors connected to a microcontroller-based system which also performs all intersection control functions [7] and Realtime Recognition and Re-identification of Vehicles from Video Data with high Re-identification [8]. But still world has traffic jam problem parallel to increasing population and vehicle number especially in big cities.

Nowadays people spent their time in traffic. It is called as "rush-hours." This time is non-productive time which stolen from people life. In addition to rush hours, the negative effect of exhaust emission occurs during waiting in the traffic jam. Finally, vehicle fuel consumption and emissions at or near street intersections are usually higher than on other street segments because the intersection frequently causes vehicles to slow, stop, accident and accelerate. [9-13]

This paper aims to simulate four-way traffic lights and green wave flow over the five junctions. It has also dynamic change of traffic signal due to traffic volume at critical intersections together with updating green wave time in every cross to safe passing of vehicles without stop. This study will be guided for problem solving of traffic jam.

\section{Simulation of Traffic Lights}

\subsection{Cycle Time Arrangement and Simulation}

Cycle time is an important parameter for organizing of traffic flow on junctions. Sometimes, this time is shared between cross randomly. When it is shared without 
numerical values of volume distribution, sometimes, vehicles on one junction, have to wait signal which let them pass even there is no vehicle on other junction. This situation makes "rush-time" and waste of money. In big cities, which has crowded junctions must be arranged the cycle of time with analyzing of traffic volume distribution.

This section includes discussion of cycle time and sharing between crosses which depends on volume distribution of vehicle as shown in Figure 1 which includes 4-way and five crosses application in an isolated area.

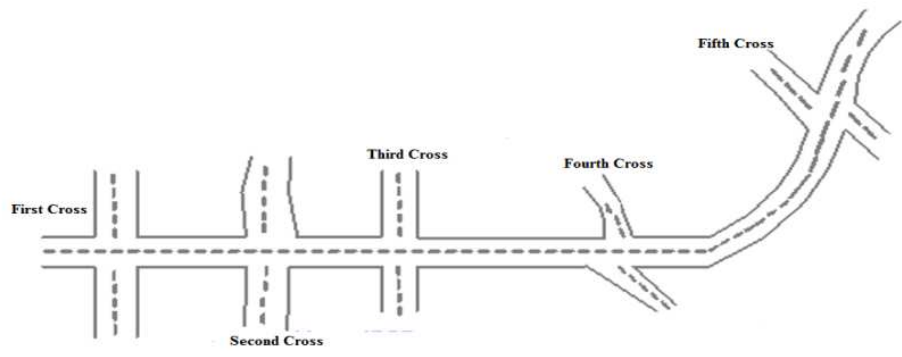

Figure 1. 4-way and Five Crosses Traffic Road in an Isolated Area

There is a relation between cycle time for signal and volume distribution on a cross;

,$\in Z^{+}$there is five cross simulation so $\operatorname{imax}=5$ and imin=1,

Cycle time without yellow signal (seconds), ( $\mathrm{Ti}-2)$.

So green signal or red signal can be calculated below,

$$
\begin{gathered}
\mathrm{Gij}=\frac{\mathrm{Vij}^{2}}{\sum_{j=1}^{2} \mathrm{Vij}_{\mathrm{ij}}} \times(T i-2) \\
R i j=(T i-2)-G i j
\end{gathered}
$$

It can be embedded to simulation of traffic light. State duration can be explained as below;

$S^{\prime}$ 'ijm=Rij seconds if $\mathrm{m}=1$ and $\mathrm{j}=1$ or $\mathrm{S}^{\prime} \mathrm{ijm}=\mathrm{Gij}+3$ seconds if $\mathrm{m}=1$ and $\mathrm{j}=2$

S'ijm=Gij-1seconds if $m=2$ and $j=1$ or $S^{\prime} i j m=R i j-$ 1 seconds if $\mathrm{m}=2$ and $\mathrm{j}=2$

This will be shown in Figure 3.

There is an unbalance time cycle due to default transition. After a while, distortion occurs during simulation. It can be easily heal by self transition in every $C i^{\prime}$ cross. This transition is used after $T i+4$ seconds on specified crosses, $C i^{\prime}$ state exits after that time and goes inside its state instantaneously. It is repeated in every cycle. The system renews itself with self transition. It will be discussed and can be shown in Figure 3.

Cycle time division includes some steps as shown in Figure 2. This figure is also representation of Matlab model of isolated area. Only first cross cycle time division is represented shown in this figure. The other four crosses will be same as shown in Figure 2. The time input firstly goes inside "Matlab function" and arranges time per one cycle equation(1) which depends on vehicles volume of whole junctions and this value is assigned to signal time for red. After that, the signal time for red goes into second "Matlab function 1" and it is recalculated then it is assigned to signal for green. Thus when it is changed cycle time, the dividing cycle time ratio between one cross to another will be always same.

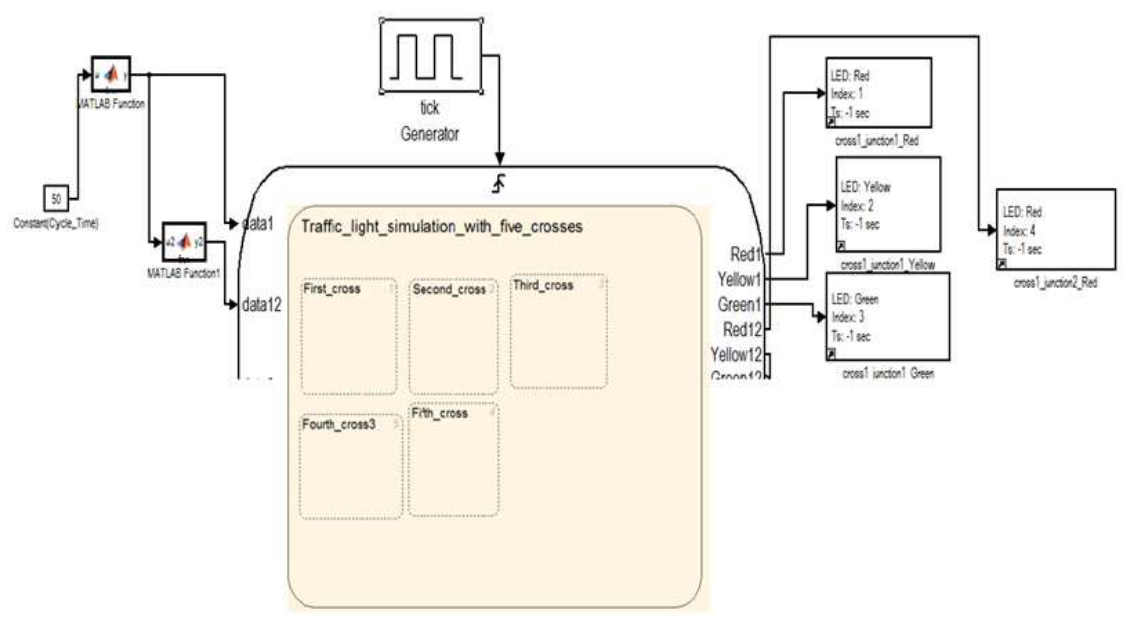

Figure 2. Picturial Representation of Main State with Sub States, Input Cycle Time and LEDs

In Figure 2 it can be also seen the representation of main states which has sub-charts. These sub-charts are working parallel when the program is executed [14].

When one of these main states, in Figure 2, is opened it can be seen new states of one junction as shown in Figure 3. It is called as state_1. This state is parallel states which make it to simulate simultaneously with other states. This state reserves one state which calls as First_cross. This 
state has two parallel states which include the situation of lights on two different junctions at one cross. First_junction_cross1 starts its default state with Stop1 lights red led, at the same time Second_junction_cross 1 starts its default state of Gol2 and it lights green led. The transitions between states depend on data_1 and data_2 parameters. After data_1-1, seconds, Go12 exits state and program runs to Stop $1 \overline{2}$ state. After one second this event, Stopl is finished and the program runs to state Readyl which has two parallel states lights Red1 and Yellowlon same junction simultaneously. After 2 seconds of entering Ready1, the first junction lets vehicle to pass. After passing data12-1 seconds, the first junction light turns to red and finishes first cycle. In every cycle, the balance of lights time gradually distortion.

It is mentioned before that after a while, the green led lights simultaneously on different junctions because of unbalance transition time between junctions due to default states. This is a big mistake but it could be fixed and healed as to create self transition in First_cross. After data1+data12+4 seconds the state of First_cross turns to its first situation like the program turns beginning situation. Every that time, it means that the program completes its one cycle and repeat this transition according as the program works. Now the system works properly.

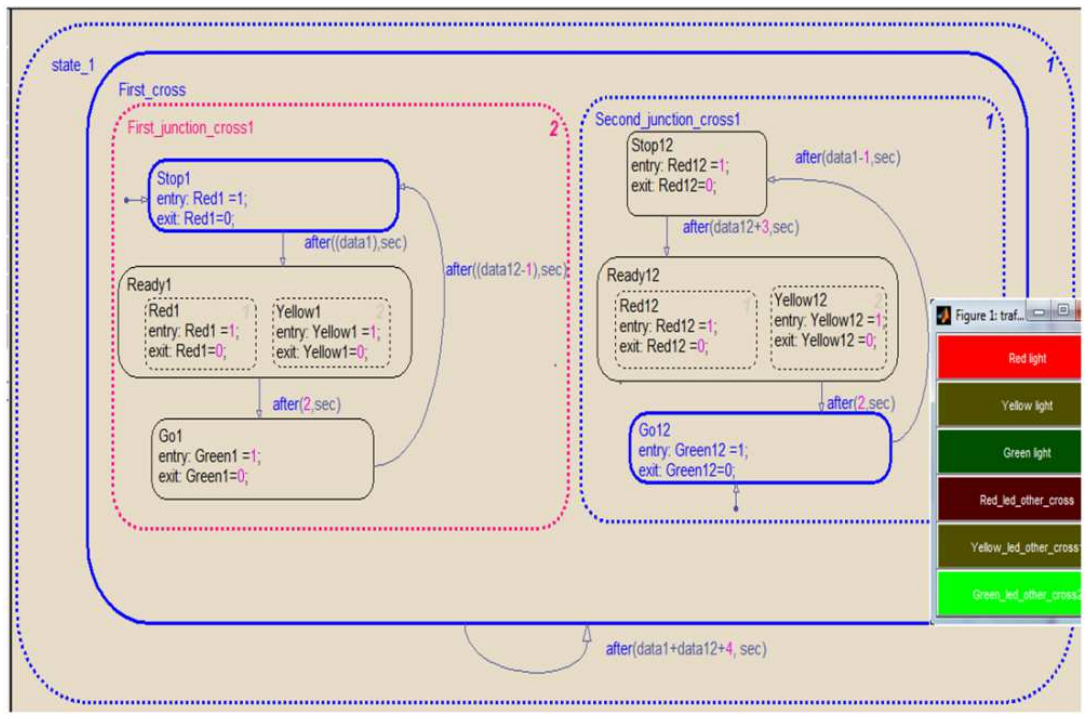

Figure 3. States of First Junction and Simulation (Day Cycle)

\subsection{Green Wave Application and Simulation}

New researchers have already suggested improving traffic globally. The new approaches make vehicles passed in the traffic without stopping under velocity constrain from one arterial to another arterials. It is called as "Green Wave". A green wave is a kind of pattern such that if vehicles travels at a certain velocity from specified junction to others, they will not hit any red lights. But the signal between specified crosses must be synchronized. There are lots of main reasons using green wave in big cities which have traffic jam problem. Traffic network congestion causes delays which add substantial costs to society and businesses on a daily basis and also increase emissions and the risk of accidents. The study mentioned above reports that in 2002 people where spending 100,000 hours in total in queues in the Greater Copenhagen road infrastructure, this corresponds to an economic loss of more than 750 million Euros [10]. This kind of problems and hazardous consequences can be reduced by optimization of green wave $[15,16]$.

There are many investigations about vehicle fuel consumption and emission at signalized intersection where signal causes vehicles slow, stop and accelerate. This situation makes vehicle consume excess fuel and produce more emission. Analytical Fuel Consumption Model was worked by The University of Texas at Austin, USA and experimental study was done which was called as TEXAS model (Traffic Experimental and Analytical Simulation model) in 1998. This model was compared with Webster (1958) who derived optimal cycle time using his empirically developed delay equation [9].

According to these studies, Green wave application is an important criterion for fuel consumption and emissions at intersections and discusses in this section.

Simulation of green wave will be discussed in this model which has five different arterials (4-way), as shown in Figure 1, are synchronized together for adapting of green wave. All crosses in this simulation can be thought run on with their own clock. As shown in Figure 4 the simulation starts with sleeping mode in the night. We can adjust sleeping mode from night to day and also from day to night.

After night_cycle seconds the state night 1 exits its state goes in new state which is day cycle. It is represented in Figure 3. When the program finishes its day cycle, the lights on junction take it sleeping mode again.

To adjust proper green wave we should start with sleeping mode. Distance and cycle of time is shown in Table 1. 
Table 1: Distance Between Crosses and Time for Green Wave Depends on Vehicle Velocity

\begin{tabular}{l|c|c|c|c|c} 
traffic cross & distance $(\mathrm{m})$ & Green wave $(\mathrm{km} / \mathrm{h})$ & vehicle velo. $(\mathrm{m} / \mathrm{s})$ & spent time $(\mathrm{sec})$ & cycle of time $(\mathrm{sec})$ \\
\hline 1st cross & & & & & 50 \\
\hline 2nd cross & 500 & 45 & 12.5 & 40 & 50 \\
\hline 3rd cross & 400 & 45 & 12.5 & 32 & 50 \\
\hline 4th cross & 300 & 45 & 12.5 & 24 & 50 \\
\hline 5th cross & 250 & 45 & 12.5 & 20 & 50 \\
\hline & & & & From starting cross to another & \\
\hline & & & & for green wave & \\
\hline
\end{tabular}

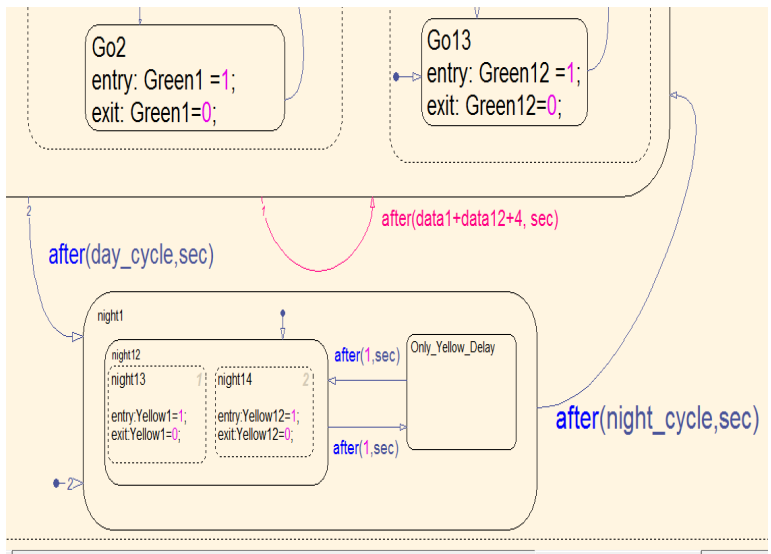

Figure 4. Sleeping Mode Cycle for First Cross

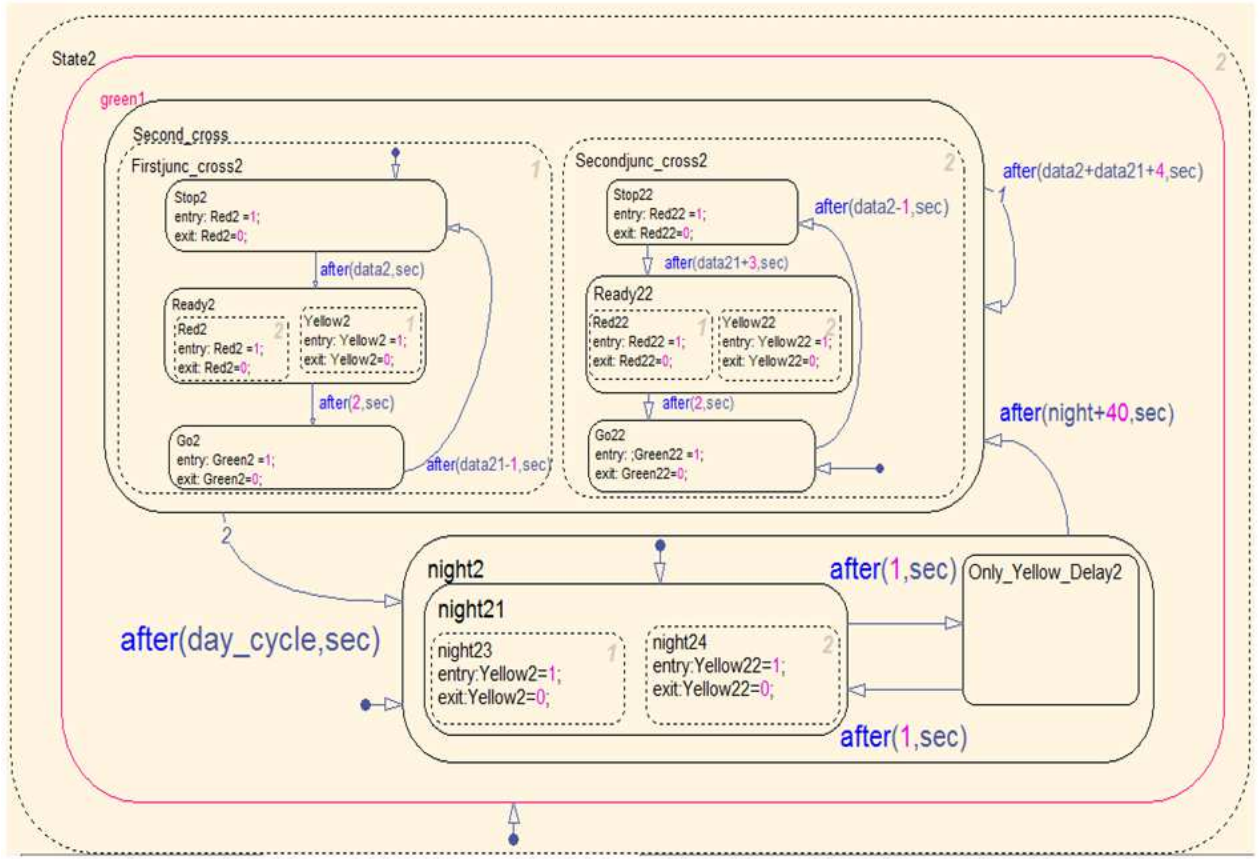

Figure 5: Sleeping Mode Cycle and Optimization of Green Wave for Second Cross

It is mentioned before that every cross uses its own clock and simulation starts from night to day state in this simulation. Normally night cycle for each crosses are same but in this simulation night cycle on specific cross is different than others for green wave. Also cycle time and vehicle velocity should be constant as shown in Table 1. Now distance is only variable parameter for synchronization of green wave. It is explained before $\mathrm{Ci}$, as number of cross. New variable is;

Distance between cross (meter), Cik, $\sum_{i=1}^{\mathrm{n}}, \mathrm{n} \in \mathrm{N}$ and $\mathrm{k}=\mathrm{i}+1$, for example when $\mathrm{i}=5$;

C56 means that distance between fifth and sixth crosses.

Spend time from one cross to other (seconds), Sik

$$
\text { Sik }=\frac{\text { Cik }}{\text { Vehicle Velocity }}
$$


It can be embedded to simulation which has five different crosses as shown in Figure 1. Crosses must be delayed to turn day cycle except first cross for green wave. For example: in Figure 6, second cross time for night cycle is night+40 seconds. Sik is equal to 40 as calculated in equation (3) shown in Table 1 and $i=1$.

When the program is executed, the simulation begins sleeping mode which let vehicles pass without red light hit at nights, and after night_cycle it is going on day cycle. The night cycle for cross one is equal to night_cycle but the rest of crosses are not if it is wanted to modify green wave, it must be set up night cycle for second cross as night_cycle +40 seconds. The day cycle starts for second cross after that time and the second cross specified junction

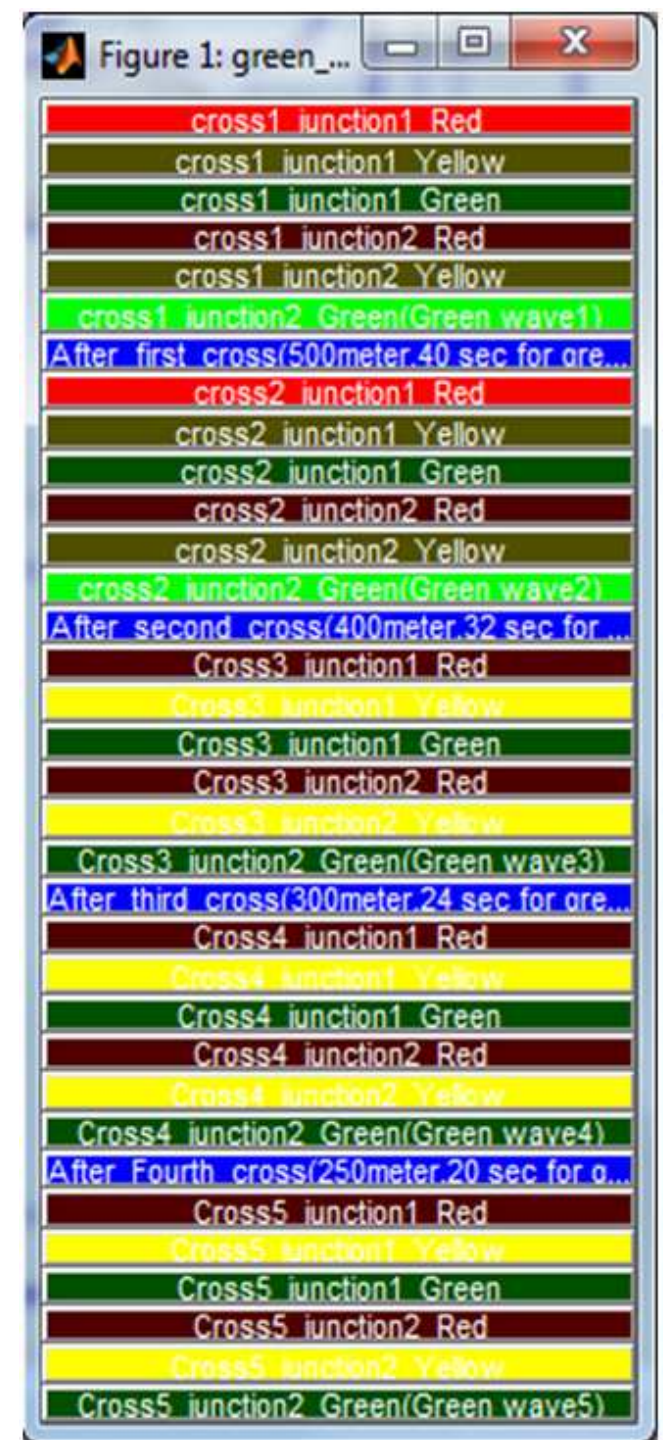

a. After night +40 seconds is joined to green wave as shown in Figure 6. We should also set up night cycle time for other crosses with adding spent time which is calculated equation (3) as shown in Table1

Green wave application is set up only second junctions of all crosses which were assumed critical. The rest of three crosses for green wave can be modified like in Figure 6 just adding green spent time in Table 1. After night +40 seconds the simulation represents lights of led as shown in Figure 7a. After night seconds, the first cross starts its day simulation. In Figure $7 \mathrm{~b}$ we can see the day simulation of all crosses after passing specified time in Table 1. And all second junctions of crosses were modified to green wave situation.

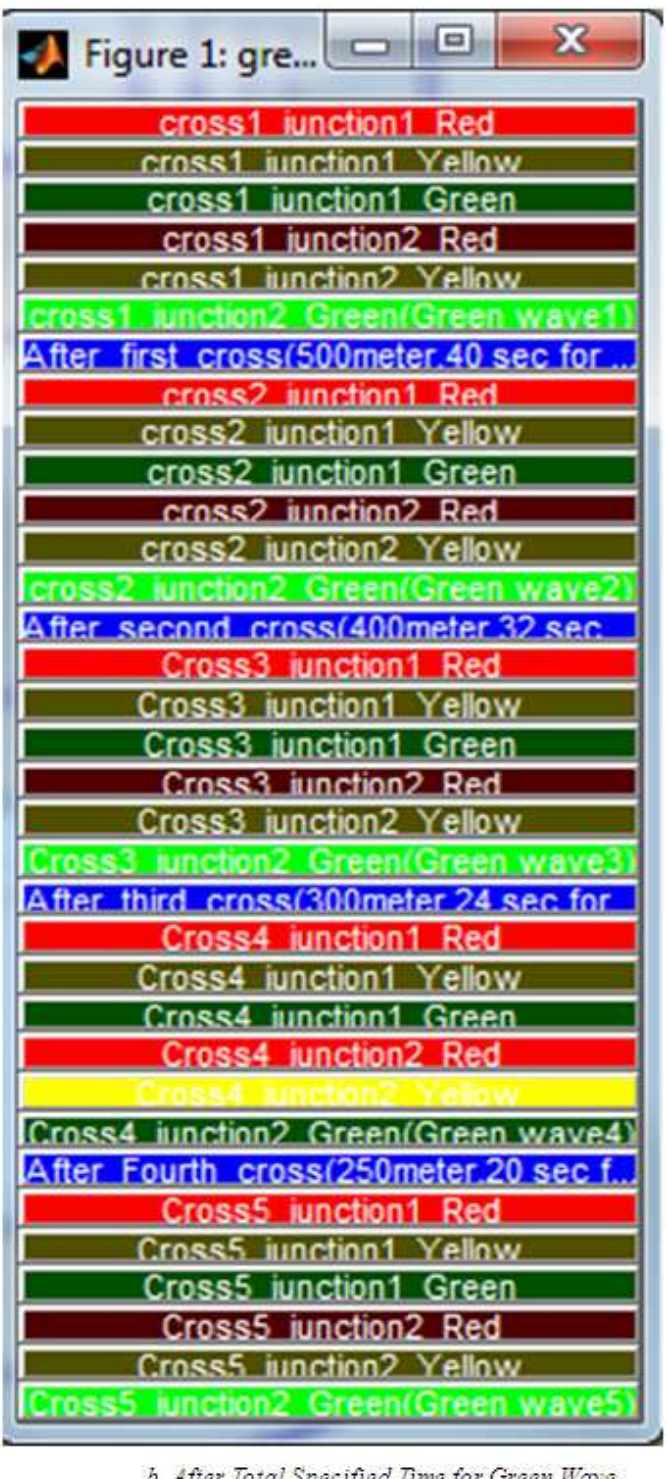

Figure 6. Simulation of LEDs for Green Wave Application

\subsection{Dynamic Change of Signal and Modified to Green Wave}

Dynamically change of signal in the traffic is an important situation over the past years in the parallel of increasing vehicle on the roads. Developing technology help to learn number of passing vehicles at intersection and getting data for processing to the next intersection on the traffic. This data can be used to modify signal for next intersections to prevent drivers from traffic jam. Some kind 
of counters are video cameras; Real-time Recognition and Re-identification of Vehicles from Video Data with high Re-identification Rate[8] and metallic mass detector, the sensing element is placed just below the road [6].

This section is about representation dynamical change of signal depends on traffic volume at one intersection and modified the simulation again depends on green wave. So, the flow of traffic and green wave mustn't be affected by changing dynamically and instantaneously.

Dynamical change should be updated specified cross. For example, first cross. New variable will be defined for representation number of vehicle on specified cross and junction shown in Figure 8. There is a slider picture called as "traffic volume at first cross."

Mathematical model of dynamic change;

Traffic volume on specified cross for dynamical change of signal, Vdyna,

Dynamic green wave time (seconds), Di; i: cross number.
In this simulation critical vehicle volume is 40 as shown in Figure 7 as "dynamic $1>40$ ". It can be also thought number of vehicle waits for green signal on specified junction.

If $V$ dyna, $i=40, \mathrm{i}=1$ then turns signal to red for other junction which lights green led and turns signal to green for this junction $(\mathrm{i}=1)$ then let vehicles pass. For green wave update; Sik should be modified which was defined before as "Spend time for green wave from one cross to other" to update green wave synchronization.

If Vdyna, $\mathrm{i}=40$ then

$$
\mathrm{Di}=\sum_{i=1}^{\mathrm{n}} \mathrm{Sik}-1, \quad \mathrm{i}: \text { number of cross }
$$

This will be used for new transition to update green wave as shown in Figure 9.

$\mathrm{D} 3=\sum_{i=1}^{3} \mathrm{Sik}-1$ means $\mathrm{S} 12+\mathrm{S} 23-1$ equal to 71 seconds as shown in transition.

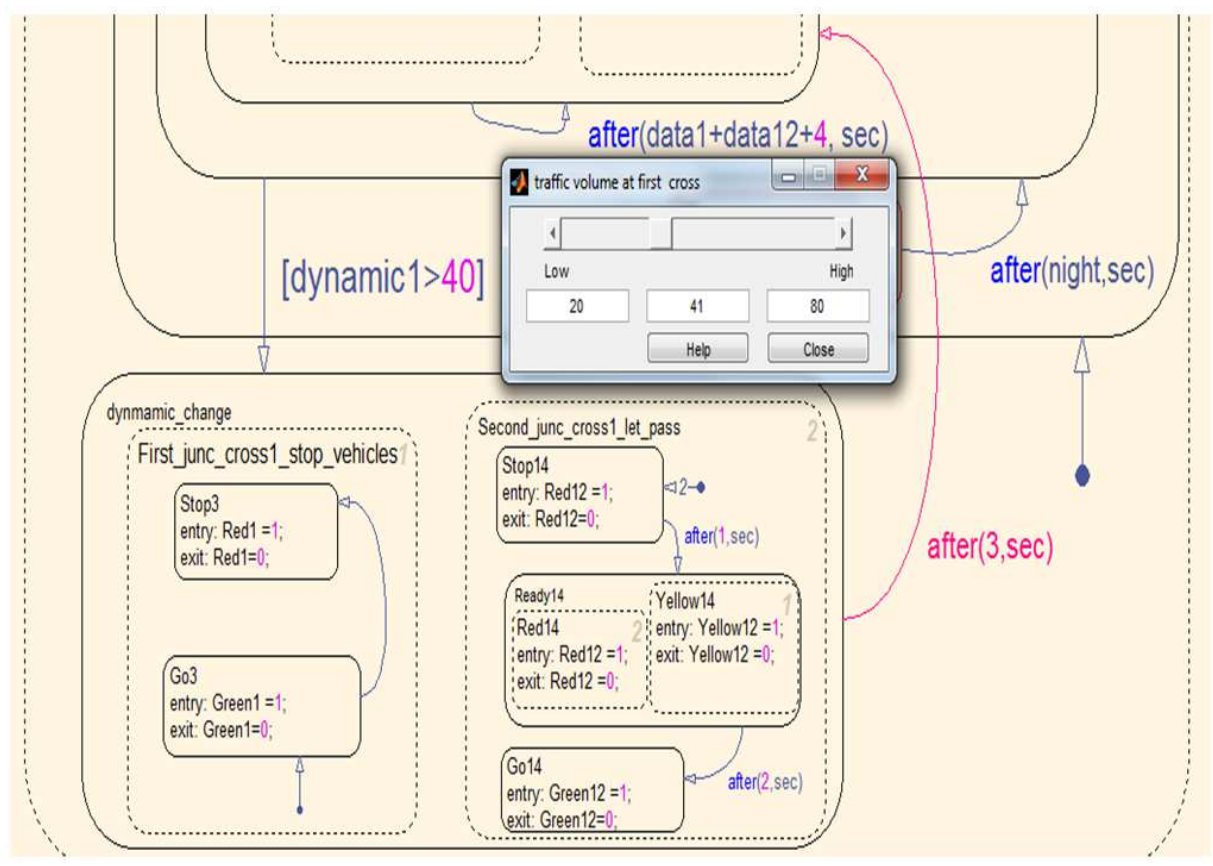

Figure 7. Representation of Dynamical Change of Traffic Volume on First Cross

Using dynamical gain box, as shown in Figure 7 , program can be thought like a counter of vehicle. It was been specified range of vehicle number before the simulation. During the simulation a junction which is specified before turns its light to red and after a while the number of vehicle at this junction increase dramatically then it will slide dynamic gain as if sending digital signal from vehicle counter machine to signal process port. This change of signal triggers states as shown in Figure 8 and let the signal turns to green at specified junction.

After change of traffic volume at first junction, the rest of junctions should be affected to modify this simulation for green wave. Figure 7 represents what would happen on third cross when traffic volume at first junction was changed.
It was discussed about sleeping mode previous section. After sleeping mode of traffic light, state of simulation works properly for green wave application. Dyna3 in Figure 8 simulates its default states parallel with main states simulation. From default state to adjust_green_wave depends only [dynamic1>40]. When traffic volume changes at first cross it means that volume is larger than 40 vehicles. Dynamic slider, in Figure 7, is moved to the right. Then the main state on first cross goes in to sub state, dynamic_change, at the same time default dyna3 on third cross go in to adjust_green_wave in Figure 8. In the sub state of first cross, first junction lights green led and second junction lights red led, then first cross' light turns red light after 1 second and second junction lights green led after 3 seconds to let vehicles pass. After 3 seconds of triggering 
dynamic gain the first cross turns to initial condition again it means that red led for first junction and green led for second junction on cross one. The rest of other main crosses don't affect this condition until specified time. They progress their duty of modifying green wave but it is not correct because of broken of green wave time with chancing signal at first cross at any time. Analyze of 3rd cross in Figure 8. Why it was specified 71 second? After 3 seconds of dynamical change 1st cross turns its initial condition. On 3rd cross to turn its initial condition takes 75 seconds. Difference seconds between these cross is 72 seconds, in Table 1, which make 3rd cross modify for green wave after 1 st cross start simulation. Preventing of accident it should be modified other crosses for example after changing traffic volume it is not known which led lights after 71 second for 3 rd cross. It is better to create if transition after specified time as shown in Figure 8. If Green $3==1$ then go into Adjust_green_cross 3 _ 1 change Go3 to Red3 for first junction and after 2 seconds change Red32 to Ready32 and 2 seconds after change Ready32 to Green32. After 4 seconds coming into day3 turn to initial condition and join to green wave. If Green 3 doesn't equal to 1 means that Green 32 led lights and go on to light green led for Green 32 until 4 second then turn to initial condition and join green wave. It can be seen that total spend time for initial condition for 3 rd cross is 75 seconds.

In summary, it be can modified the conditions and states for other crosses and make sure for green wave synchronize.

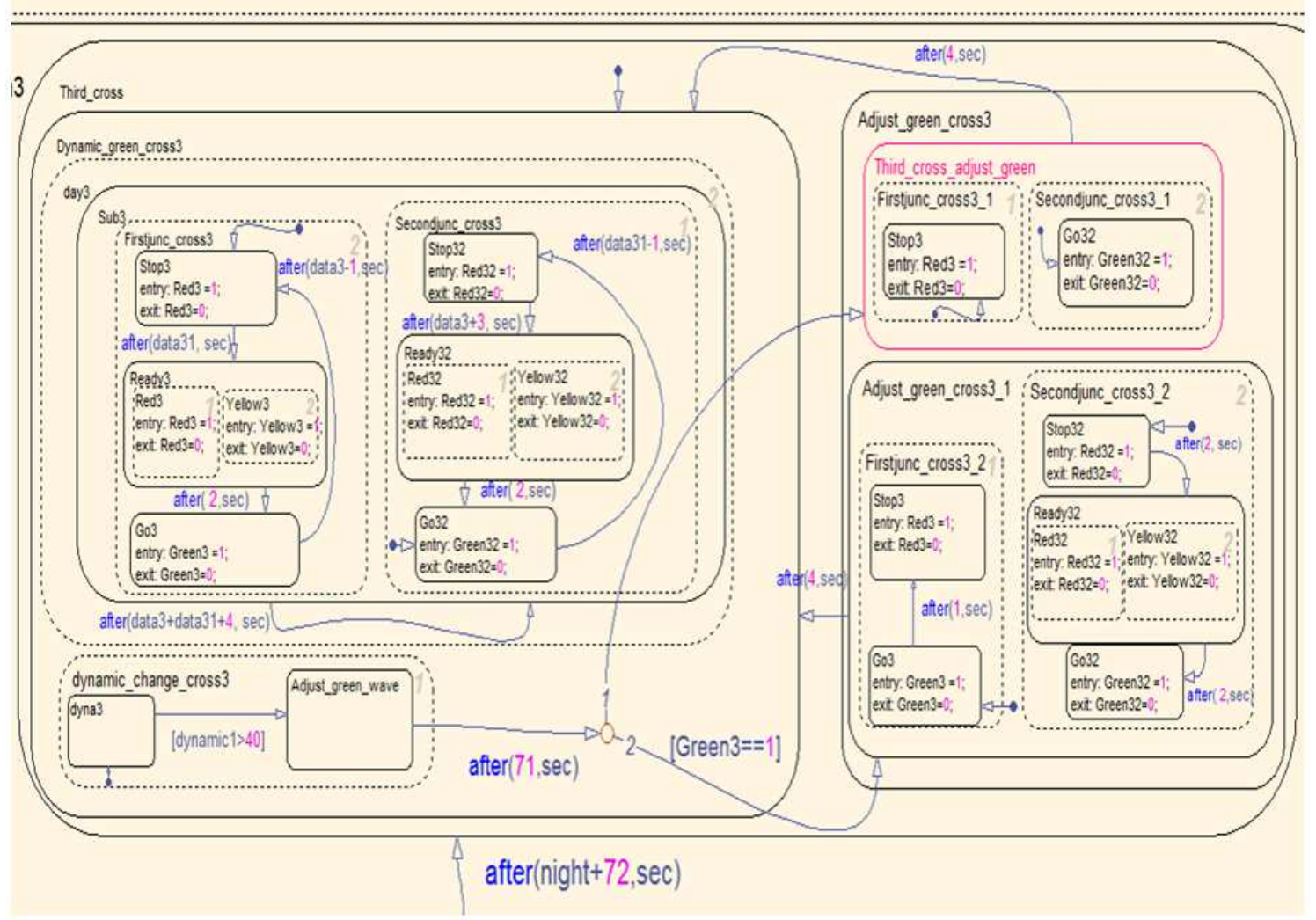

Figure 8: States Condition to Modify Green Wave on Second Cross after Dynamically Change of Specified Intersection

\section{Conclusion}

This study has presented a model for simulation of traffic control and signal. Application and simulation of green wave and dynamical change of signal depends on traffic volume at essential intersection.

In the section of simulation of traffic light, it was firstly discussed about importance of cycle time. The cycle time of one intersection is one of the most important parameters for proper planning and flowing of traffic. Also in this section, it was defined input cycle time and let it changed depending on traffic volume at intersection via using Matlab function as shown in Figure 3. Then it was mentioned about five crosses simulation which execute concurrently in away using parallel states. In this section we also demonstrated simulation of one cross in hierarchy as shown in Figure 4. After that it was dealt with how green wave application works and mentioned about its benefits in addition to them it was mentioned how people's life is affected in a good way by green wave application. Then it was discussed how to modify our state flow chart to green wave.

Finally, it was adjusted state flow chart to change signal dynamically at specified intersection and learnt how to overcome problems due to dynamical change of signal at any time causes to spoil green wave cycle.

As a result, Matlab/Simulink/Stateflow suits well to representation, simulation and adjustment of dynamic changes. This study may be guided for development of future projects about traffic signal and control systems. 


\section{Nomenclature}

i : Specified cross, $i \in Z^{+}$

$\mathrm{j}$ : number of junction on specified cross.

: Number of cross

: Volume distribution on a junction weekly or monthly; $j=1$ first junction, $\mathrm{j}=2$ second junction

: Cycle time in each cross (seconds)

: Signal time for red light (seconds)

: Signal time for green light (seconds)

$C^{\prime} \mathrm{i}$ : Cross in simulation

$S^{\prime}$ ijm : State duration in simulation (seconds); $\mathrm{m}=1$ for red light, $\mathrm{m}=2$ for green light

: Distance between crosses (meter); $\mathrm{k}=\mathrm{i}+1$

: Spent time for green wave one cross to other cross

Vdyna, : Traffic volume on specified cross for dynamical change of signal

: Dynamic green wave time (seconds)

\section{References}

[1] http://www.ideafinder.com/history/inventions/trafficlight.ht $\mathrm{m}$

[2] J. Niittymäki, M. Pursula. Signal Control Using Fuzzy Logic; Fuzzy Sets and Systems, Vol. 116(1), 2000, pp. 1122

[3] A.S.M. Rahman, N.T. Ratrout. Review of the Fuzzy Logic Based Approach in Traffic Signal Control: Prospects in Saudi; Journal of Transportation Systems Engineering and Information Technology, Vol. 9(5), 2009, pp. 58-70

[4] I. Kosonen. Multi-Agent Fuzzy Signal Control Based on Real-Time Simulation; Transportation Research Part C: Emerging Technologies, Vol. 11(5), 2003, pp. 389-403

[5] W. M. Wey. Model Formulation and Solution Algorithm of Traffic Signal Control in an Urban Network; Computers Environment and Urban Systems, Vol. 24(4), 2000, pp.355378
[6] M. J. Caruso, L. S. Withanawasam. Vehicle Detection and Compass Applications using AMR Magnetic Sensors; Sensors Expo Proceedings, May 1999, pp.477-489.

[7] K. Tavladakis, N. C. Voulgaris. Development of Autonomous Adaptive Traffic Control System in ESIT'99; The European Symposium on Intelligent Techniques, 1999.

[8] Rate, Woesler, Richard. Real-time Recognition and Reidentification of Vehicles from Video Data with high Reidentification; 8th World Multiconference on Systemics, 2004, pp.347-352

[9] T. Y. Liao, R. B. Machemehl. Development of an Aggregate Fuel Consumption Model for Signalized Intersections; Transportation Research Board of the National Academies, Vol. 16(41), 1998, pp.9-18

[10] A. Warberg, J. Larseny, R. M. Jorgensen. Green Wave Traffic Optimization- A survey Informatics and Mathematical Modelling; Technical University of Denmark, DTU, 2008, pp.1-23

[11] H. Rakha, Y. Ding, Impact of stops on vehicle fuel consumption and emissions; Journal of Transportation Engineering, Vol.129 (1), 2003, pp.23-32,

[12] I. D. Greenwood C. R. Bennett. The Effects of Traffic Congestion on Fuel Consumption; Road \& Transport Research, Vol. 5, No. 2, June 1996, pp. 18-31.

[13] C.Gershenson. Self-organizing Traffic Lights; Complex Systems, Vol.16 (1), 2005, pp.29-53

[14] Stateflow User's Guide the Math Works, Inc.

[15] http://www.wired.com/wiredscience/2010/09/traffic-lightsadapt/

[16] D.Greenwood, B. Burdiliak, I. Trencansky, H. Armbruster. Green Wave Distributed Traffic Intersection Control; Proceedings of the 8th International Conference on Autonomous Agents and Multiagent Systems, Vol.2, 2009, pp.1413-1414. 